\title{
Produção Científica dos Pesquisadores da Área de Pediatria no $\mathrm{CNPq}$

\author{
Scientific Research in Pediatrics Produced \\ at the $\mathrm{CNPq}$
}

\author{
Eduardo Gonçalves \\ Maria Ivanilde Pereira Santos ${ }^{I}$ \\ Bruna Tupinambá MaiaII \\ Rairana Chiara Silveira BrandãoIII \\ Eduardo Araújo Oliveira ${ }^{I V}$ \\ Hercílio Martelli Júnior ${ }^{I}$
}

\author{
PALAVRAS-CHAVE \\ - Indicadores de Produção \\ Científica; \\ - Educação Médica; \\ - Pesquisadores; \\ - Pediatria; \\ - Ciências da Saúde.
}

\section{KEYWORDS}

- Indicators of Scientific Production;

- Medical Education;

- Research Personnel;

- Pediatrics;

- Health Sciences.

\begin{abstract}
Recebido em: 13/05/2014
\end{abstract}
Aprovado em: 31/07/2014

REVISTA BRASILEIRA DE EDUCAÇÃO MÉDICA

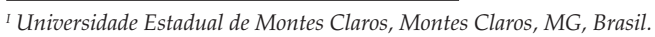

${ }^{I I}$ Faculdades Integradas Pitágoras de Montes Claros - FIP-Moc, Montes Claros, MG, Brasil.

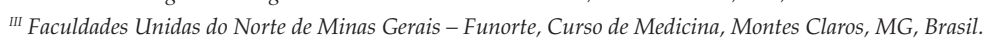

${ }^{\text {IV }}$ Universidade Federal de Minas Gerais - UFMG, Belo Horizonte, MG, Brasil. 


\section{INTRODUÇÃO}

A produção científica brasileira é a $13^{\mathrm{a}}$ do mundo, respondendo por 2,69\% da produção mundial e superando países como Suíça (1,83\%), Suécia (1,65\%), Holanda (2,53\%) e Rússia $(2,53 \%)^{1}$. No Brasil, a produção científica, avaliada pelo número de publicações indexadas no Institute for Scientific Information (ISI), vem apresentando um importante incremento nos últimos $a^{2}{ }^{2}$. De acordo com a National Science Indicators (NSI) da Thomson Reuters Scientific INC e SJR, em 2012, as publicações realizadas por pesquisadores brasileiros em periódicos indexados saltaram de 32.100 em 2009 para 53.083 em 2012.

A avaliação sistemática de pesquisadores, periódicos, universidades e instituições de pesquisa constitui uma atividade que, embora controversa, tem sido relevante para cientistas e gestores ${ }^{4}$. Além disso, as agências de fomento precisam de avaliações sistemáticas para otimizar alocações de recursos e definir estratégias para os órgãos de pesquisa, possibilitando reestruturar a pesquisa em domínios específicos ou aumentar a produtividade em pesquisa no País ${ }^{5}$. Neste contexto, assume importância a denominada bolsa de produtividade em pesquisa (PQ) do Conselho Nacional de Desenvolvimento Científico e Tecnológico (CNPq), incentivo criado na década de 1970. Essa bolsa foi concebida como uma forma de incentivo aos pesquisadores detentores de título de doutor e de destacada produção científica em suas áreas de atuação, para valorizar seu trabalho frente a seus pares. Sendo assim, o perfil dos atuais bolsistas PQ torna-se de interesse para a comunidade científica ${ }^{6}$.

Vários estudos têm analisado o perfil e a produção científica dos pesquisadores do CNPq em diversos campos do conhecimento ${ }^{6-10}$. Recentemente, foi avaliado o perfil dos PQ da área de Pediatria referente ao período 2006-2008 ${ }^{11}$. O presente estudo descreve as características demográficas e a produção acadêmica dos pesquisadores $\mathrm{PQ}$ em Medicina do CNPq, cuja área de atuação é a Pediatria.

\section{METODOLOGIA}

Realizou-se um estudo de métrica, do tipo transversal e analítico. Inicialmente foi construído um banco de dados com 529 pesquisadores da área da Medicina registrados como bolsistas $\mathrm{PQ}$ do $\mathrm{CNPq}$, de acordo com a relação publicada pela agência federal de fomento à pesquisa ${ }^{3}$. Em seguida, foram selecionados os pesquisadores que atuam especificamente na área de Pediatria. Foram avaliados todos os pesquisadores da Pediatria com bolsas ativas durante o triênio 2010-2012. Foram excluídos do estudo os pesquisadores que se encontravam com bolsas suspensas, como nos casos de realização de pósdoutorado ${ }^{12-14}$.
A área da Pediatria foi considerada como área de atuação quando esta informação específica foi disponibilizada pelo pesquisador no currículo Lattes (CL). Quando tal informação não estava contida no $C L$, foi analisada pelos autores deste estudo a produção científica do pesquisador nos últimos três anos e atribuída uma área na qual havia predominância de temas publicados e/ou orientados. Em casos específicos de atuação em subáreas bem definidas, como, por exemplo, Nefrologia Pediátrica, o pesquisador foi inserido na área da Pediatria, e a subárea de atuação foi considerada como uma variável à parte. Seguindo essa metodologia, foram identificados 47 pesquisadores $\mathrm{PQ} / \mathrm{CNPq}$ da Medicina com atuação específica na área da Pediatria.

A partir da identificação dos pesquisadores bolsistas PQ da Pediatria, foram consultados os CL de todos os pesquisadores considerando as categorias existentes no CNPq: sênior, 1A, 1B, 1C, 1D e 2. Com base nos CL disponibilizados publicamente na Plataforma Lattes $(\mathrm{CNPq})^{3}$, foi construído um banco de dados por meio do programa SPSS ${ }^{\circledR}$ para Windows, versão 18.0, com informações relativas à distribuição dos PQ por categoria, distribuição geográfica e institucional, tempo de conclusão do doutorado, produção científica (artigos científicos, livros e capítulos de livros), formação de recursos humanos (orientação de estudantes de iniciação científica, de mestrado e de doutorado) e produção de patente. Não foi identificado pesquisador PQ da categoria sênior na aérea da Pediatria no triênio 2010-2012. Para as análises estatísticas foram consideradas a produção científica e as orientações durante o triênio 2010-2012.

Foi também pesquisada a base de dados $\operatorname{Sicapes}^{3}$ e analisada a classificação Qualis dos periódicos nos quais os pesquisadores PQ da Pediatria têm publicado. Tal classificação é categorizada em A1, A2, B1, B2, B3, B4 e C. O nome científico do pesquisador e os periódicos utilizados nessa investigação foram aqueles fornecidos no CL. Após a construção do banco de dados por meio do programa estatístico SPSS versão 18.0 para Windows, realizou-se a análise estatística descritiva e univariada dos dados obtidos.

\section{RESULTADOS}

$\mathrm{O}$ universo de pesquisadores $\mathrm{PQ}$ do $\mathrm{CNPq}$ da área da Medicina no triênio 2010-2012 correspondeu a 529 bolsistas, todos ativos. Destes, 47 (8,8\%) foram identificados como pesquisadores da Pediatria, sendo 31 (66\%) do gênero masculino e 16 (34\%) do feminino (Gráfico 1). No que se refere aos níveis dos pesquisadores da Pediatria, houve predominância daqueles pertencentes ao nível 2 (51\%), seguidos dos níveis 1D (17\%), 1C $(9 \%), 1 \mathrm{~A}$ e $1 \mathrm{~B}$, ambos correspondendo a $4 \%$ do total. 


\section{GRÁFICO 1}

Distribuição dos bolsistas de produtividade em pesquisa da área da Pediatria segundo gênero e categorização do CNPq $(\mathrm{n}=47)$

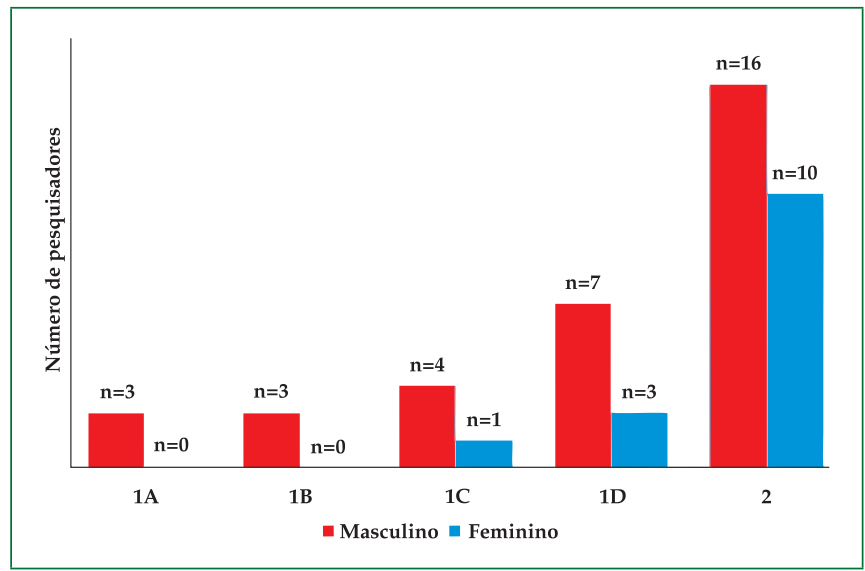

GrÁfico 2

Distribuição dos pesquisadores da área da Pediatria por instituição de origem e categorização da bolsa do $\mathrm{CNPq}(\mathrm{n}=47)$

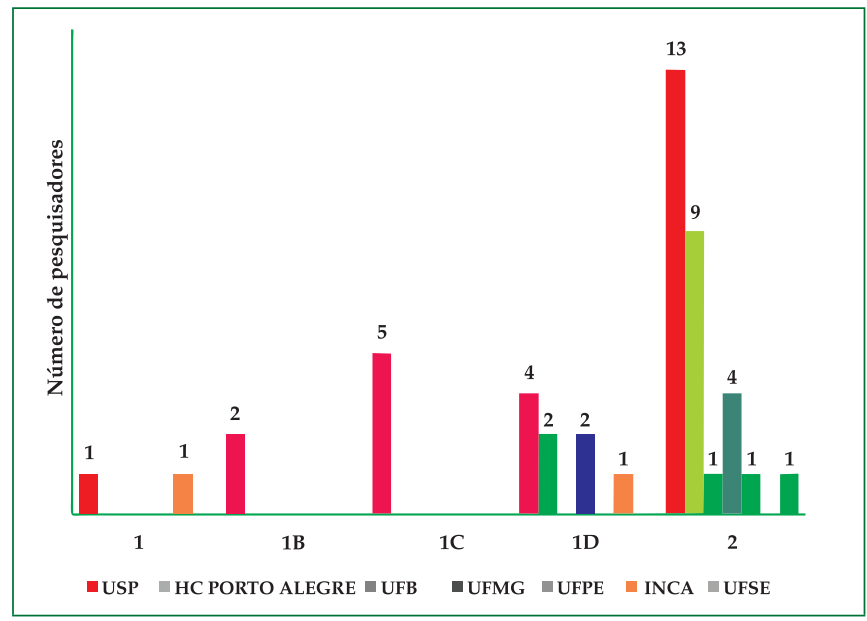

Os pesquisadores PQ da Pediatria estão distribuídos em sete Estados da federação (São Paulo, Rio Grande do Sul, Bahia, Minas Gerais, Pernambuco, Rio de Janeiro e Sergipe), com maior concentração nas regiões Sudeste (68\%) e Sul (23\%). Em último lugar encontra-se a Região Nordeste, que abriga 9\% $(n=4)$ dos pesquisadores PQ desta área. Dos 32 bolsistas filiados a instituições da Região Sudeste, 24 (51\%) pertencem ao Estado de São Paulo, enquanto 11 (24\%) se encontram no Rio de Janeiro. Em terceiro lugar está Minas Gerais, que abriga $13 \%(n=6)$ dos bolsistas. A distribuição dos 47 pesquisadores por categoria da bolsa está sumarizada no Gráfico 2.
A produção científica dos PQ da Pediatria no triênio 20102012 abrangeu o total de 1.174 artigos publicados, sendo que $73 \%$ foram escritos por pesquisadores do gênero masculino e os $27 \%$ restantes pelo gênero feminino. Deste número, $24 \%$ foram veiculados em periódicos com classificação Qualis B2 e 23\% em B1 (Tabela 1).

Os pesquisadores PQ da Pediatria se distribuem em 15 diferentes instituições do País. As instituições que mais publicaram artigos foram: USP (36,3\%), Unifesp (11,6\%), UFMG (11,5\%) e Unicamp (8,5\%). As outras instituições com publicações foram: Hospital das Clínicas de Porto Alegre, Instituto Nacional do Câncer, Instituto de Cardiologia do Rio Grande do Sul, PUC-RS, UFBA, UFPE, UFRGS, UFRJ, UFSE, UFSJ, Unesp. Com relação ao vínculo institucional dos pesquisadores, a Tabela 1 mostra a relação do pesquisador, a instituição e o nível da bolsa.

Essa tabela também indica a porcentagem dos artigos científicos publicados segundo instituição de origem do pesquisador e Qualis dos periódicos. A maioria dos artigos $274(23,3 \%)$ foi publicada em periódicos com Qualis B2, e os pesquisadores do nível 2 foram responsáveis pela publicação de 537 (45,7\%) deste total. Já a Tabela 2 mostra a distribuição dos artigos científicos publicados segundo gênero do pesquisador e Qualis dos periódicos. No que se refere aos artigos publicados por Estados e regiões, verificou-se que São Paulo foi responsável por 689 (58,7\%) destas publicações, seguido do Rio Grande do Sul (195 publicações; 16,6\%) e Minas Gerais (171 publicações; 14,6\%). A Região Sudeste produziu 911 artigos (77,6\%), o Sul 195 (16,6\%) e o Nordeste $68(5,8 \%)$.

Ainda no indicador correspondente à produção científica, constatou-se a publicação de 27 livros no triênio analisado. Os capítulos de livros publicados foram em número de 217 , e, destes, $59 \%$ foram produzidos por autores do gênero masculino. Todos os livros, 97\% dos capítulos de livro, 99\% das orientações de iniciação científica e $92 \%$ das orientações de mestrado e doutorado, bem como a patente encontrada foram de autoria de pesquisadores $\mathrm{PQ}$ que tiveram como sede de doutorado o Brasil.

Outro importante parâmetro na atividade científica dos pesquisadores bolsistas é a formação de recursos humanos, ou seja, a orientação de alunos de iniciação científica, de mestrado e de doutorado. As orientações de iniciação científica no triênio estudado foram 215 , sendo $66 \%$ realizadas pelo gênero masculino e $34 \%$ pelo gênero feminino. As orientações de mestrado somaram 297, e as de doutorado corresponderam ao total de 254. No triênio analisado, foi encontrada uma patente produzida por um pesquisador da Pediatria. 


\begin{tabular}{|c|c|c|c|c|c|c|c|c|c|c|}
\hline Distribuição dos artig & $\begin{array}{r}\text { científic } \\
\text { da }\end{array}$ & s public & $\begin{array}{r}\text { TAI } \\
\text { Idos seg } \\
\text { Q Quali }\end{array}$ & $\begin{array}{l}\text { LA } 1 \\
\text { Indo a ir } \\
\text { Capes d }\end{array}$ & stituição & $\begin{array}{l}\text { de orige } \\
\text { icos }\end{array}$ & n do pe & uisado & da área & \\
\hline Instituição de origem & A1 (\%) & A2 (\%) & B1 (\%) & B2 (\%) & B3 (\%) & B4 (\%) & B5 (\%) & $\mathrm{C}(\%)$ & $\begin{array}{l}\text { Sem } \\
\text { Qualis }\end{array}$ & Total \\
\hline Hospital das Clínicas de Porto Alegre & 2,7 & 3,2 & 1,5 & 2,6 & 0,0 & 4,4 & 0,0 & 0,0 & 3,5 & 2,1 \\
\hline Instituto Nacional do Câncer & 4,4 & 1,9 & 2,2 & 1,5 & 2,7 & 2,2 & 0,0 & 0,0 & 1,8 & 2,1 \\
\hline $\begin{array}{l}\text { Instituto de Cardiologia do } \\
\text { Rio Grande do Sul }\end{array}$ & 1,1 & 0,0 & 3,0 & 0,4 & 0,0 & 0,0 & 0,9 & 0,0 & 0,0 & 1,0 \\
\hline PUC-RS & 10,9 & 7,0 & 6,3 & 5,5 & 6,8 & 8,9 & 8,3 & 25,0 & 5,3 & 7,2 \\
\hline UFBA & 1,1 & 5,7 & 2,2 & 3,6 & 1,4 & 0,0 & 0,9 & 25,0 & 1,8 & 2,6 \\
\hline UFMG & 8,2 & 8,2 & 14,8 & 9,5 & 6,8 & 24,4 & 20,2 & 0,0 & 5,3 & 11,5 \\
\hline UFPE & 0,5 & 3,2 & 0,4 & 3,6 & 0,0 & 0,0 & 0,9 & 50,0 & 1,8 & 1,8 \\
\hline UFRGS & 6,6 & 3,8 & 7,7 & 5,5 & 5,5 & 4,4 & 8,3 & 0,0 & 7,0 & 6,2 \\
\hline UFRJ & 2,7 & 0,6 & 1,5 & 2,9 & 2,7 & 6,7 & 0,0 & 0,0 & 5,3 & 2,2 \\
\hline UFSE & 0,5 & 2,5 & 0,7 & 2,2 & 1,4 & 0,0 & 1,8 & 0,0 & 0,0 & 1,4 \\
\hline UFSJ & 4,9 & 3,8 & 1,1 & 5,8 & 0,0 & 0,0 & 1,8 & 0,0 & 0,0 & 3,1 \\
\hline Unesp & 6,0 & 1,3 & 1,8 & 0,7 & 2,7 & 0,0 & 2,8 & 0,0 & 3,5 & 2,3 \\
\hline Unicamp & 2,7 & 7,6 & 5,9 & 16,1 & 17,8 & 11,1 & 0,0 & 0,0 & 8,8 & 8,5 \\
\hline Unifesp & 4,9 & 7,6 & 7,7 & 13,9 & 15,1 & 15,6 & 33,0 & 0,0 & 3,5 & 11,6 \\
\hline USP & 42,6 & 43,7 & 43,2 & 26,3 & 37,0 & 22,2 & 21,1 & 0,0 & 52,6 & 36,3 \\
\hline Total & 100 & 100 & 100 & 100 & 100 & 100 & 100 & 100 & 100 & 100 \\
\hline
\end{tabular}

\begin{tabular}{|c|c|c|c|c|c|c|c|c|c|c|}
\hline \multicolumn{11}{|c|}{$\begin{array}{c}\text { TABELA } 2 \\
\text { Distribuição dos artigos científicos publicados pelos pesquisadores da área da Pediatria segundo gênero } \\
\text { e Qualis-Capes dos periódicos }\end{array}$} \\
\hline Gênero & A1 (\%) & A2 (\%) & B1 (\%) & B2 (\%) & B3 (\%) & B4 (\%) & B5 (\%) & C (\%) & $\begin{array}{l}\text { Sem } \\
\text { Qualis }\end{array}$ & Total \\
\hline Masculino & 75 & 68 & 68 & 77 & 74 & 78 & 72 & 75 & 75 & 73 \\
\hline Feminino & 25 & 32 & 32 & 23 & 26 & 22 & 28 & 25 & 25 & 27 \\
\hline Total & 100 & 100 & 100 & 100 & 100 & 100 & 100 & 100 & 100 & 100 \\
\hline
\end{tabular}

O número de orientações de iniciação científica diminui discretamente de 2010 para 2011 (66 para 61) e aumenta em 2012 ( $n=91)$. As orientações de mestrado cresceram no triênio analisado, verificando-se o total de 71 estudantes orientados em 2010, 97 em 2011 e 130 em 2012. A quantidade de orientações de doutorado também aumentou no decorrer dos três anos, sendo identificadas 55 em 2010, 75 em 2011 e 124 em 2012. A Tabela 3 indica a produção de livros, capítulos de livros, formação de recursos humanos e patentes, segundo o nível do pesquisador.

Com relação às instituições e Estados de origem do pesquisador, chama atenção o desempenho dos Estados de São
Paulo, Rio Grande do Sul e Minas Gerais. A UFRGS publicou mais livros $(12 ; 44 \%)$, e a USP publicou a maioria dos capítulos de livros $(87 ; 40,1 \%)$ e das orientações de doutorado (64; $25,2 \%$ ). A UFMG, por sua vez, foi responsável por 42 iniciações científicas (19,5\%), e a PUC-RS por 57 orientações de mestrado (19,2\%). O Estado de São Paulo abrigou a maioria das publicações de capítulos de livros (157; 72,4\%), 70 orientações de iniciação cientifica $(32,6 \%)$ e 114 orientações de doutorado (44,9\%). O Estado do Rio Grande do Sul foi responsável por 13 livros publicados (48,1\%) e 106 orientações de mestrado $(35,7 \%)$. A única patente encontrada no triênio pertence ao Estado de Pernambuco. 


\begin{tabular}{|c|c|c|c|c|c|c|}
\hline Distribuição d & $\begin{array}{l}\text { ros, cap } \\
\text { produz }\end{array}$ & $\begin{array}{l}\text { de livros } \\
\text { elos pesc }\end{array}$ & $\begin{array}{c}\text { TAвELA } 3 \\
\text { rientações de inicia } \\
\text { sadores da Pediatri }\end{array}$ & $\begin{array}{l}\text { ientífica, de } \\
\text { undo nível }\end{array}$ & $\begin{array}{l}\text { do, de douto } \\
\text { uisador }\end{array}$ & atentes \\
\hline Nível Pesquisador & Livros & $\begin{array}{l}\text { Capítulo } \\
\text { Livro }\end{array}$ & $\begin{array}{c}\text { Orientação } \\
\text { Iniciação Científica }\end{array}$ & $\begin{array}{l}\text { Orientação } \\
\text { Mestrado }\end{array}$ & $\begin{array}{l}\text { Orientação } \\
\text { Doutorado }\end{array}$ & Patentes \\
\hline Não especificou & 5 & 67 & 15 & 44 & 22 & 0 \\
\hline $1 \mathrm{~A}$ & 0 & 6 & 2 & 16 & 20 & 0 \\
\hline $1 \mathrm{~B}$ & 2 & 23 & 24 & 4 & 23 & 0 \\
\hline $1 \mathrm{C}$ & 2 & 24 & 5 & 13 & 11 & 0 \\
\hline $1 \mathrm{D}$ & 14 & 20 & 26 & 69 & 46 & 0 \\
\hline 2 & 4 & 77 & 143 & 151 & 132 & 1 \\
\hline Total & 27 & 217 & 215 & 297 & 254 & 1 \\
\hline
\end{tabular}

\section{DISCUSSÃO}

O estudo mostrou que existe uma concentração de pesquisadores bolsistas do CNPq da área da Pediatria no Brasil nas regiões Sudeste e Sul. Esta concentração é relatada por outros estudos que avaliam o desempenho de pesquisadores de outras áreas do conhecimento. A principal explicação para este fato está associada à presença na Região Sudeste de tradicionais centros formadores que desenvolvem pesquisas voltadas para a saúde da criança e do adolescente e também à presença de instituições que historicamente apresentam alta produtividade nesta área ${ }^{11-15}$.

Do ponto de vista estadual, os resultados deste estudo apontam uma concentração tanto de pesquisadores quanto da produção científica da Pediatria brasileira no Estado de São Paulo, corroborando os resultados de outros estudos envolvendo outras áreas do conhecimento ${ }^{13-15}$. Nesta mesma direção, estudos recentes mostram que na área da Medicina apenas três Estados brasileiros são responsáveis por aproximadamente $90 \%$ dos pesquisadores, sendo que mais da metade está concentrada no Estado de São Paulo ${ }^{11-13}$. Reforçando a concentração paulista em relação ao número de pesquisadores, estudos mostram que a média nacional do número de bolsistas de produtividade em pesquisa por habitantes é de 2,14 bolsistas em Medicina por milhão de habitantes ${ }^{13}$. Entretanto, apenas três Estados apresentam uma razão bolsistas/milhão de habitantes acima da média nacional (São Paulo, Rio Grande do Sul e Rio de Janeiro) ${ }^{6}$.

Os achados deste estudo revelam também uma concentração de pesquisadores e da produção na área da Pediatria em poucas instituições do País. O estudo chama atenção para três universidades paulistas (USP, Unifesp e Unesp), que respon- dem por percentuais significativos da pesquisa em Pediatria no universo dos pesquisadores bolsistas do CNPq, ressaltando-se que estas instituições são consideradas importantes centros formadores e produtores de conhecimento científico na área da Pediatria. A pujança destas instituições paulistas é bastante relatada na atualidade, sobretudo por investigações que têm como universo pesquisadores do CNPq de diversas áreas do conhecimento ${ }^{6,13}$. Sobre esta questão, estudos apontam que sete instituições brasileiras são responsáveis por aproximadamente $80 \%$ dos pesquisadores do País, destacando a USP $(51,0 \%)$ e a Unifesp $(17 \%)^{9}$.

Um resultado deste estudo que merece destaque se refere ao aumento significativo da produção científica da Pediatria no triênio 2010-2012. Em média, os pesquisadores do CNPq na área da Pediatria duplicaram o número de artigos publicados, comparando-se a média anual em toda a carreira e nos últimos três anos. Entretanto, o aumento da produção científica é uma realidade brasileira, fato observado também em outras áreas do conhecimento, como Odontologia, Saúde Pública e Fisioterapia ${ }^{6-8,10}$. Corroborando esta informação sobre o aumento da produção na Pediatria, estudos que tiveram como universo pesquisadores do CNPq da área de Medicina como um todo apontam que apenas cinco subáreas tiveram a produção aumentada em mais de duas vezes no período 2004-2008, quando comparadas as médias de publicação ao longo da carreira: Pediatria, Cardiologia, Oftalmologia, Clínica Médica e Pneumologia ${ }^{13}$. Esse incremento quantitativo na produção científica na Pediatria reflete o aumento geral da produção científica brasileira e, possivelmente, resulta dos vários mecanismos indutores estabelecidos pelas diversas agências nacionais de fomento à pesquisa ${ }^{14}$. 
O presente estudo demonstra que, além do aumento quantitativo das publicações brasileiras na área da Pediatria, a qualidade das publicações também melhorou, embora a maioria das publicações seja classificada como B2 e B1. Segundo os documentos da Capes referentes à área Medicina II, na qual se enquadram os programas de pós-graduação em Pediatria, os periódicos são classificados como A1 quando o Fator de Impacto (FI) é superior ou igual a 4, e como A2, quando o FI se situa entre 2,8 e $3,9^{15}$.

Considerando a participação do pesquisador bolsista do CNPq nas orientações de iniciação científica, mestrado e doutorado, o estudo aponta uma importante contribuição dos pesquisadores da Pediatria, na medida em que todas estas modalidades de orientações aumentaram no triênio analisado. É importante destacar que este aumento também foi encontrado nos estudos que avaliam outra área ${ }^{16}$.

$\mathrm{O}$ estudo aponta um aumento das publicações em periódicos indexados, tanto nacionais quanto internacionais. Quanto ao Qualis dos periódicos em que os pesquisadores PQ da Pediatria publicam seus artigos, embora 23\% estejam publicando em periódicos B1 e 24\% em B2, é importante destacar que um percentual significativo destes pesquisadores $(29 \%)$ tem publicado seus artigos em periódicos com classificação Qualis A (A1 e A2). Este fato pode estar associado à preocupação recente dos pesquisadores com a visibilidade de suas publicações e com as exigências atuais das agências de fomento.

Muitos fatores levam a uma promoção da produção científica brasileira ${ }^{16}$. Sobre esta questão, ressalta-se que as agências de fomento e comitês institucionais responsáveis por eleger candidatos para posições acadêmicas (como bolsistas $\mathrm{PQ}$, por exemplo) normalmente baseiam suas decisões em critérios como número de publicações científicas em periódicos de prestígio internacional e desempenho individual dos pesquisadores. A Capes, para conceituar os programas nacionais de pós-graduação brasileiros, prioriza não só a quantidade, mas também a qualidade dos artigos publicados ${ }^{17,18}$. Além do mais, a competição entre pesquisadores com bolsa de produtividade em pesquisa incentiva tanto a formação de novos pesquisadores como a publicação de artigos em periódicos de impacto $^{13}$ e maior oportunidade de contatos entre os profissionais $^{19,20}$.

\section{REFERÊNCIAS}

1. Petherick A. High hopes for Brazilian science. Nature 2010;465:674-5.

2. Brasil. Ministério da Ciência e Tecnologia. Indicadores nacionais de ciência e tecnologia (C\&T): comparações internacionais - produção científica. 2009 [citado 28 Feverei- ro 2014]. Disponível em: http://www.mct.gov.br/index. php/content/view/9231.html

3. Brasil. Ministério da Ciência e Tecnologia. MdCe. Indicadores nacionais de ciência e tecnologia (C\&T): comparações internacionais - produção científica. 2012 [citado 16 Abril 2014]. Disponível em: http://www.mct.gov.br/index. php/content/view/5710.html.

4. Randic M. Citations versus limitations of citations: beyond Hirsch index. Scientometrics 2009;80(3):811-20.

5. Moed HF. New developments in the use of citation analysis in research evaluation. Arch Immunol Ther Exp (Warsz) $2009 ; 57(1): 13-8$.

6. Santos NCF, Candido LFO, Kuppens CL. Produtividade em pesquisa do CNPq: análise do perfil dos pesquisadores da química. Quimica Nova 2010;33 (2):489-95.

7. Barata RB, Goldbaum M. A profile of researchers in public health with productivity grants from the Brazilian National Research Council (CNPq). Cad Saúde Pública 2003;19(6):1863-76.

8. Cavalcante RA, Barbosa DR, Bonan PRF, Pires MBO, Martelli-Junior H. Perfil dos pesquisadores da área de odontologia no Conselho Nacional de Desenvolvimento Científico e Tecnológico (CNPq). Rev Bras Epidemiol 2008;11(1):106-13.

9. Mendes PHC, Martelli DR, Souza WP, Filho SQ, Martelli-Junior H. Perfil dos pesquisadores bolsistas de produtividade científica na medicina no $\mathrm{CNPq}$, Brasil. Rev Bras Educ Méd 2010;34(4):535-41.

10. Santos SMC, Lima LS, Martelli DRB, Martelli Junior H. Perfil dos pesquisadores da Saúde Coletiva no Conselho Nacional de Desenvolvimento Científico e Tecnológico. Physis Revista de Saúde Coletiva 2009;19(3):761-75.

11. Oliveira MCL, Martelli DRB, Pinheiro SV, Miranda DM, Quirino IG, Leite BGL, et al. Profile and scientific production of Brazilian National Council of Technological and Scientific Development researchers in Pediatrics. Rev. Paul Pediatr 2013;31(3):278-84.

12. Oliveira EA, Colosimo EA, Martelli DR, Quirino IG, Oliveira MC, Lima LS et al. Comparison of Brazilian researchers in clinical medicine: are criteria for ranking well-adjusted? Scientometrics 2011;90:429-43.

13. Martelli-Junior H, Martelli DR, Quirino IG, Oliveira MC, Lima LS, Oliveira EA. CNPq researchers in medicine: a comparative study of research areas. Rev Assoc Med Bras 2010;56(4):478-83

14. Panaretos J, Malesios CC. Assessing scientific research performance and impact with single indices. Scientometrics 2009;81:635-70. 
15. Brasil. Ministério da Educação. CAPES [homepage na Internet]. Brasília: Comunicado nº 001/2012 - área de Medicina II: atualização do webqualis da área [citado em $30 \mathrm{dez}$ 2013]. Disponível em: http:/ /www.capes.gov.br/images / Qualis_-_Medicina_II.pdf

16. Sturmer G, Viero CCM, Silveira MN, Lukrafka JL, Plentz RDM. Profile and scientific output analysis of physical therapy researchers with research productivity fellowship from the Brazilian National Council for Scientific and Technological Development. Braz J Phys Ther 2013; 17(1):41-48.

17. Deheinzelin D, Caramelli B. Scientific production, post-graduate education and Revista da Associação Médica Brasileira. Rev Assoc Med Bras 2007;53(6):471-2.

18. Qualis Periódicos. 2012. Disponível em: http:/ / qualis.capes.gov.br/webqualis/principal.seam [Capturado em: 10 fev. 2014]

19. Guimarães JA. As razões para o avanço da produção científica brasileira [CAPES web site]; 2012. Disponível em: http: / / www.capes.gov.br/servicos / sala-de-imprensa / artigos / 4720-as-razoes-para-o-avanco-da-producao-cientifica brasileira. [Acessed 12 February 2014].

20. Thomson Reuters. Journal of Citations Reports; 2013 [Thomson Reuters web site]. Disponível em: http:// thomsonreuters.com/products_services/science/science_ products/a-z/journal_citation_reports/.Accessed. [Acessed 19 December 2013].

\section{CONTRIBUIÇÃO DOS AUTORES}

Eduardo Gonçalves participou no estudo e redação e revisão do tema. Maria Ivanilde Pereira Santos elaborou a análise dos dados e tabulação estatística dos mesmos, com redação da metodologia do artigo científico. Bruna Tupinambá Maia participou da seleção dos artigos para elaboração do referencial teórico, desenho do estudo e revisão final do artigo científico. Rairana Chiara Silveira Brandão participou da seleção dos artigos para elaboração do referencial teórico, desenho do estudo e revisão final do artigo científico. Eduardo Araújo Olivieira participou da seleção dos artigos para elaboração do referencial teórico, desenho do estudo e revisão final do artigo científico. Hercílio Martelli participou no desenho do estudo e redação e revisão crítica do tema.

\section{CONFLITO DE INTERESSES}

Não houve conflito de interesses.

\section{ENDEREÇO PARA CORRESPONDÊNCIA}

Eduardo Gonçalves

Rua Gabriel Passos, 130/401 - Centro

Montes Claros - Minas Gerais - Brasil

CEP: 39400-112

E-mail: eduardo.goncalves2000@yahoo.com 